\title{
High CD44 Immunoexpression Correlates with Poor Overall Survival: Assessing the Role of Cancer Stem Cell Markers in Oral Squamous Cell Carcinoma Patients from the High-Risk Population of Pakistan
}

\author{
Yumna Adnan $\mathbb{D}^{\mathbb{D}},{ }^{1,2}$ S. M. Adnan Ali $\mathbb{D}^{1},{ }^{1}$ Hasnain A. Farooqui $\mathbb{D}^{1},{ }^{1}$ Hammad A. Kayani $\mathbb{D}^{2}{ }^{2}$ \\ Romana Idrees $\mathbb{D}^{3}{ }^{3}$ and M. Sohail Awan $\mathbb{D I}^{4}$ \\ ${ }^{1}$ Office of Academia and Research in Surgery, Department of Surgery, Aga Khan University Hospital, Karachi, Pakistan \\ ${ }^{2}$ Department of Biosciences, Faculty of Life Sciences, Shaheed Zulfikar Ali Bhutto Institute of Science and Technology, \\ Karachi, Pakistan \\ ${ }^{3}$ Section of Histopathology, Department of Pathology and Laboratory Medicine, Aga Khan University Hospital, Karachi, Pakistan \\ ${ }^{4}$ Section of Otolaryngology, Head and Neck Surgery, Department of Surgery, Aga Khan University Hospital, Karachi, Pakistan
}

Correspondence should be addressed to S. M. Adnan Ali; syed.adnan@aku.edu

Received 27 October 2021; Accepted 11 February 2022; Published 7 March 2022

Academic Editor: C. H. Yip

Copyright ( 2022 Yumna Adnan et al. This is an open access article distributed under the Creative Commons Attribution License, which permits unrestricted use, distribution, and reproduction in any medium, provided the original work is properly cited.

Oral squamous cell carcinoma (OSCC) is a top-ranked cancer in the Pakistani population, and patient survival has remained unchanged at $\sim 50 \%$ for several decades. Recent advances have claimed that a subset of tumour cells, called cancer stem cells (CSCs), are responsible for tumour progression, treatment resistance, and metastasis, which leads to a poor prognosis. This study investigated the impact of CSC markers expression on overall survival (OS) and disease-free survival (DFS) of OSCC patients. Materials and Methods. Immunohistochemistry was used to evaluate CD44, CD133, L1CAM, and SOX2 expression in a wellcharacterized cohort of 100 Pakistani patients with primary treatment naïve OSCC. The immunoreactivity for each marker was correlated with patient clinicopathologic characteristics, oral cancer risk chewing habits, and survival. The minimum follow-up time for all patients was five years, and survival estimates were calculated using the Kaplan-Meier method and Cox proportional hazards model. Results. In this cohort of 100 patients, there were 57 males and 43 females. The median OS and DFS time durations observed were 64 and 52.5 months, respectively. Positive expression for CD44, CD133, L1CAM, and SOX2 was observed in 33\%, $23 \%, 41 \%$, and $63 \%$ of patients. High CD44 expression correlated with decreased OS $(P=0.047)$ but did not influence DFS. However, CD133, L1CAM, and SOX2 had no effect on either OS or DFS. Tonsils, nodal involvement, and AJCC stage were independent predictors of worse OS and DFS both. Conclusion. Of the CSC markers investigated here, only CD44 was a predictor for poor OS. CD44 was also associated with advanced AJCC and T stages. Interestingly, CD133 was significantly lower in patients who habitually consumed oral cancer risk factors.

\section{Introduction}

Oral cavity cancer is one of the leading causes of cancerrelated death in South Central Asia, including Pakistan. It is the first and second most common cancer in Pakistani males and females, respectively, and has the second-highest rate of oral cavity cancers worldwide, thus continuing to be a major public health crisis and a significant hurdle in improving life expectancy $[1,2]$.

The rationale for the high incidence of oral cavity cancers in Pakistan, and South Asia in general, is the frequent, persistent, and prevalent use of substances classified as oral cancer risk factors. These include betel quid, areca nut, alcohol, smoking, and smokeless tobacco. 
Despite recent advances in imaging technology and treatment modalities, the last few decades have seen limited improvement in the survival rate of oral cancer. At our centre, we have observed approximately $40-50 \%$ of patients survive five years following diagnosis [3].

More than $90 \%$ of oral cancers are oral squamous cell carcinomas (OSCC), arising from the squamous epithelia of the oral cavity. The cancer stem cell hypothesis states that cancer stem cells (CSCs) are a subpopulation of multipotent cells at the core of a tumour that is responsible for tumour differentiation, tumour maintenance, and spread to other sites [4]. CSCs are believed to evade or be resistant to conventional treatment and thus can generate new tumour cells that are genetically identical to the parent tumour. This self-renewal ability of CSCs leads to disease recurrence and treatment failure. The role of CSCs has not been fully elucidated in OSCC [5].

It may be that subpopulations of CSCs at the core of OSCC tumours are the source of tumour regrowth. To improve patient survival, there is a need to design therapies targeted towards identifying and eradicating this subpopulation of self-renewing cells. The identification of CSCs is made easier by detecting the increased expression of a panel of CSC markers present on their surfaces and within. Such CSCs markers include CD44, CD133, L1CAM, and SOX2.

CD44 is a cell surface glycoprotein that regulates cell proliferation, adhesion, migration, and invasion in CSCs. Increased CD44 expression has been noted in multiple cancers such as pancreas, stomach, colon, lung, breast, prostate, salivary glands, and head and neck, among others, and has been linked to worse prognosis [6]. In OSCC, the role of CD44 in predicting prognosis is debatable as conflicting results have been reported [7, 8].

Similarly, CD133 (also known as Prominin-1) is another cell surface glycoprotein identified in hematopoietic and progenitor cells. CD133 is responsible for growth, differentiation, and cell motility and is believed to cause tumour relapse and progression towards malignancy. It has been investigated as a possible prognostic factor for melanoma, thyroid carcinoma, prostate carcinoma, retinoblastoma, brain tumours, leukaemia, renal tumours pancreatic tumours, and oral cancer $[9,10]$. However, in the case of OSCC, the prognostic impact of CD133 has not been fully validated as conflicting evidence exists.

Another factor that is critical for the maintenance and self-regeneration of stem cells is Sox2. Sox 2 is a transcription factor modulating the expression of several genes essential for the maintenance of the embryonic stem cell phenotype. In cancer, Sox 2 protein expression has been linked with a worse prognosis as it promotes drug resistance, metastasis, survival, and proliferation [11]. For OSCC, Sox 2 expression is a controversial marker considering that some studies have reported Sox2 to be linked to lymph node metastasis and poor survival, while others have found increased Sox2 expression to improve prognosis $[12,13]$.

L1CAM is a neuronal cell adhesion molecule that has been studied mainly for its role in the nervous system. Following its role in cell motility and plasticity, L1CAM has been studied in multiple cancers and is considered a negative prognostic factor in endometrial, ovarian, breast, gastric, colon, pancreatic, kidney, non-small cell lung cancer, and melanoma [14]. According to the available literature on PubMed, only one study has investigated the role of L1CAM in OSCC and found that it was correlated with poor histologic differentiation and higher invasion [15]. However, no studies have correlated the expression of L1CAM with the survival of OSCC patients.

The objective of this study was to evaluate the protein expression of CD44, CD133, L1CAM, and SOX2 and correlate their expression with risk habits, clinicopathologic factors, and overall and disease-free survival in a high-risk, resource-constrained oral cavity cancer population.

\section{Materials and Methods}

The Aga Khan University Hospital (AKUH) is a Joint Commission International (JCI), and College of American Pathologists (CAP) accredited largest tertiary-care academic medical centre situated in Karachi. It serves as the preferred referral centre for cancer patients of all socioeconomic backgrounds from all over the country.

Patients had consented to participation and had complete clinicopathological information. All patient information was retrieved from the hospital's medical records and clinic follow-ups. The minimum follow-up time for all patients was 60 months. Overall survival (OS) was taken as the number of months from date of diagnosis until last known status (if alive) or date of death. Disease-free survival (DFS) was taken as the number of months from the date of surgery until recurrence or if no recurrence then until the last follow-up (if alive) or death. Ethical approval was obtained from the Ethical Review Committee of AKUH (ERC\# 2020-0392-14105).

2.1. Sample Size Calculation. This was a retrospective cohort study comprising 100 OSCC patients who had been diagnosed and treated in the years January 1991-December 2015. The sample size calculation for this study was performed on Open Epi software (https://www.openepi.com/SampleSize/ SSCohort.htm). According to the calculations, a sample size of 100 was deemed sufficient. An anticipated frequency of expression of CSC markers among OSCC patients ranging from $10.2 \%$ for CD44, 5.8\% for CD133, and 7\% for SOX2 [16-18] was used with a $90 \%$ level of significance, $5 \%$ precision, and design effect of 1 .

2.2. Immunohistochemistry Performance. Before immunohistochemistry (IHC) performance, haematoxylin and eosin ( $\mathrm{H} \& \mathrm{E})$ stained slides of all tumour specimens were reviewed to confirm tumour content and tissue adequacy. IHC was performed manually. Formalin-fixed paraffin-embedded (FFPE) blocks were sectioned using a semiautomatic rotary microtome (pfm Rotary 3005E, pfm medical, Germany). Four-micrometre-thick tissue sections were transferred to a floating water bath to remove wrinkles and taken onto glass slides (FLEX IHC Microscope Slides, K8020, Dako, Denmark). Deparaffinization was performed for $30 \mathrm{~min}$ at $56^{\circ} \mathrm{C}$ 
in an oven, followed by dipping in xylene for $2 \mathrm{~min}$. Slides were then rehydrated using water-ethanol serial dilutions $(100 \%, 90 \%, 70 \%$, and $50 \%)$ with a final rinse in deionized water. The EnVision FLEX, High pH (Link) system (K8000221, Dako, Denmark) was used for IHC staining according to the manufacturer's recommendations. To unmask the antigen of interest, target retrieval was performed by immersing slides in high $\mathrm{pH}$ target retrieval solution (K8004, Dako, Denmark) for $30 \mathrm{~min}$ in a water bath heated at $90-95^{\circ} \mathrm{C}$. Following retrieval, slides were dipped in peroxidase blocking reagent (S2023, Dako, Denmark) to inhibit the activity of endogenous peroxidase. Following each step, slides were washed with Tris buffer saline + Tween 20 (wash buffer, S3006, Dako, Denmark). Sections were incubated in the primary antibody (CD44, CD133, L1CAM, and SOX2) according to their respective conditions. Table 1 lists the primary antibody information including clone, company, dilutions, and incubation times. The primary antibody was rinsed off with wash buffer, and the slides were treated with secondary antibody EnVision/HRP (labelledpolymer rabbit/mouse, Dako, Denmark) and incubated for another $30 \mathrm{~min}$. To visualize the antigen-antibody conjugate, $\mathrm{DAB}+$ chromogen (Dako, Denmark) was applied for $4 \mathrm{~min}$ and slides were dipped in haematoxylin (CS70030, Dako, Denmark) for $30 \mathrm{~s}$ for counterstaining. Specimens were dehydrated in a water-ethanol graded series $(50 \%, 70 \%, 90 \%$, and $100 \%$ ) and mounted with cover slides using toluene-free mounting medium (Dako, Denmark). Experimental controls were run in each batch. A previously known positive specimen for each antibody (according to the manufacturer's recommendation) was selected (Table 1) as positive control, and a slide stained with saline instead of primary antibody served as the negative control.

\subsection{Immunohistochemistry Evaluation and Scoring. Slides} were observed under a light microscope (Nikon, Japan). Two independent observers (SMAA and RI) blinded to the patient history scored the slides. At least 200 cells in 5-10 different fields using a 20x lens were observed prior to scoring. The selection of the first field was subjective, while the remaining fields were selected systematically to cover the entire tumour specimen. A scoping view of the entire slide was taken at first glance, and the areas with the highest staining were selected for review as the first field. Following this, the slide was first observed in a horizontal manner and then in a vertical manner to observe the entire specimen and then assign scoring. The scoring of immunopositive expression was performed as summarized in Table 2.

2.4. Statistical Analysis. Statistical analysis was performed using Statistical Package for Social Sciences (SPSS) version 19 (IBM, USA). The expression of CD44, CD133, L1CAM, and SOX2 were correlated with patient demographics, clinical, pathological, and survival data. Patients were considered censored observation if they were alive at the time of last follow-up (for OS analysis) or were disease-free (for DFS analysis). Kaplan-Meier curves were drawn for OS and DFS analysis and compared using log-rank statistics.
Cross-tabulations and logistic regression were run to correlate factors with markers expression and compared using the chi-square test or Fisher's exact test as appropriate. Odds ratios (OR) were reported with a $95 \%$ confidence interval (CI). Univariate Cox regression analysis was performed to evaluate the effect of markers expression and other factors on OS and DFS. Hazard ratios (HR) as estimates of relative risk were reported with $95 \% \mathrm{CI}$. All $P$ values were two-sided and significant if $<0.05$.

\section{Results}

3.1. Patient Characteristics. The study cohort comprised 57 males and 43 females with a female:male ratio of 1:1.33. The mean age of patients was $51.42, \mathrm{SD} \pm 13.33$, while the median age was 50 years. Eighty-two patients were $\geq 40$ years of age, while ages for all participants ranged from 20 to 78 years. All patients underwent surgery for primary tumour resection. Some patients received additional treatment in the form of chemotherapy (8\%), radiotherapy (65\%), or palliative care (4\%). Complete patient characteristics are available in Table 3.

3.2. CD44 Expression. CD44 immunohistochemical expression was observed as dark brown exclusively membranous staining (Figure 1(a)). CD44 positive expression was observed in the tumour cores of all patients and in the basal layer, which was expected since most epithelial stem cells are in the basal layer of the oral mucosal lining. CD44 expression was increased in the invasive front of the tissue and was present in all poorly differentiated tumours. High CD44 expression was seen in 33\% of specimens, while the remaining 67\% were classified as low CD44 expression. Although a greater number of patients with low CD44 expression were $\geq 40$ years of age, this difference was not statistically significant $(P=0.09)$.

Upon correlation of CD44 protein expression with patient clinicopathologic characteristics, it was seen that CD44-high patients had significantly advanced American Joint Committee on Cancer (AJCC) stage and T stage tumours (Table 4). Patients that were AJCC stage III had high CD44 expression $(P=0.036)$ as well as those with tumour size T3 $(P=0.007)$. Curiously, CD44 expression was also higher in patients that had floor of the mouth $(71 \%)$ as a secondary site of tumour $(P=0.038)$.

3.3. CD133 Expression. Cell membranous and cytoplasmic dark brown staining was seen in CD133 positive specimens (Figure 1(b)). CD133 positivity was observed in the plasma membrane protrusions in the tumour core cells and on the invasive front. There were 23 specimens positive for CD133 expression, while 77 were negative. Out of the 23 positive samples, 2 (9\%) had a strong expression; $6(26 \%)$ had moderate; and 15 (65\%) had mild expression. A large group of patients $\geq 40$ years of age tested negative for CD133 expression, but this did not translate to statistical significance $(P=0.077)$. 
TABLE 1: IHC protocol and antibody details.

\begin{tabular}{|c|c|c|c|c|c|c|c|c|}
\hline $\begin{array}{l}\text { S. } \\
\text { no. }\end{array}$ & Antibody & $\begin{array}{c}\text { Clone/ } \\
\text { product code }\end{array}$ & $\begin{array}{l}\text { Source, } \\
\text { clonality }\end{array}$ & Company & $\begin{array}{c}\text { Antibody } \\
\text { dilution }\end{array}$ & $\begin{array}{c}\text { Antibody } \\
\text { incubation }\end{array}$ & Positive control & Cellular location \\
\hline 1. & CD133 & EPR16508 & $\begin{array}{c}\text { Rabbit, } \\
\text { monoclonal }\end{array}$ & Abcam, UK & $1: 1,000$ & $40 \mathrm{~min}$ & $\begin{array}{l}\text { Glioblastoma } \\
\text { multiforme }\end{array}$ & $\begin{array}{c}\text { Cell membranous and } \\
\text { cytoplasmic }\end{array}$ \\
\hline 2. & $\mathrm{CD} 44$ & DF1485 & $\begin{array}{c}\text { Mouse, } \\
\text { monoclonal }\end{array}$ & $\begin{array}{l}\text { Dako, } \\
\text { Denmark }\end{array}$ & $1: 40$ & $40 \mathrm{~min}$ & $\begin{array}{l}\text { Glioblastoma } \\
\text { multiforme }\end{array}$ & Cell membranous \\
\hline 3. & L1CAM & EPR18750 & $\begin{array}{l}\text { Rabbit, } \\
\text { monoclonal }\end{array}$ & Abcam, UK & $1: 500$ & $40 \mathrm{~min}$ & Normal kidney & Cell membranous \\
\hline 4. & SOX2 & ab97959 & $\begin{array}{l}\text { Rabbit, } \\
\text { polyclonal }\end{array}$ & Abcam, UK & $1: 250$ & $40 \mathrm{~min}$ & $\begin{array}{l}\text { Glioblastoma } \\
\text { multiforme }\end{array}$ & Nuclear \\
\hline
\end{tabular}

TABLE 2: Immunoreactivity scoring criteria for all antibodies.

\begin{tabular}{|c|c|c|c|c|c|}
\hline Antibody & No. of positive cells & Type of staining & Scoring & $\begin{array}{c}\text { For statistical } \\
\text { analysis }\end{array}$ & References \\
\hline \multirow[t]{4}{*}{ CD133 } & 0 & - & Negative $(0)$ & Negative & {$[35]$} \\
\hline & $<30 \%$ & - & Weak $(+/ 1)$ & Positive & \\
\hline & $30-60 \%$ & - & Moderate $(++/ 2)$ & Positive & \\
\hline & $>60 \%$ & - & Strong $(+++/ 3)$ & Positive & \\
\hline \multirow[t]{5}{*}{$\mathrm{CD} 44$} & $\leq 10 \%$ cells & Weakly stained & Negative (0) & Negative & {$[36]$} \\
\hline & $11-30 \%$ cells & Weakly stained & 1 (low) & Low & \\
\hline & $\begin{array}{c}>30 \% \text { weakly or }<30 \% \\
\text { moderately stained }\end{array}$ & & 2 (low) & Low & \\
\hline & $30 \%-60 \%$ & Moderately stained & 3 (high) & High & \\
\hline & $>60 \%$ & $\begin{array}{c}\text { Moderately or strongly } \\
\text { stained }\end{array}$ & 4 (high) & High & \\
\hline \multirow[t]{5}{*}{ L1CAM } & $0 \%=0$ & None $=0$ & $\begin{array}{c}\text { Intensity } \times \% \text { of positive } \\
\text { cells }=\text { score }\end{array}$ & & {$[37]$} \\
\hline & $<10 \%=1$ & Weak $=1$ & $0-2=$ negative & Negative & \\
\hline & $10-50 \%=2$ & Moderate $=2$ & $3-4=$ weakly positive & Positive & \\
\hline & $51-80 \%=3$ & Strong $=3$ & $6-8=$ moderately positive & Positive & \\
\hline & $>80 \%=4$ & & $9-12=$ strong & Positive & \\
\hline \multirow[t]{4}{*}{ SOX2 } & $<10 \%$ & - & Negative $(0)$ & Negative & {$[38]$} \\
\hline & $10-50 \%$ & - & Weak $(+/ 1)$ & Positive & \\
\hline & $50-90 \%$ & - & Moderate $(++/ 2)$ & Positive & \\
\hline & $>90 \%$ & - & Strong $(+++/ 3)$ & Positive & \\
\hline
\end{tabular}

An interesting observation was that chewing/smoking habits and the nature of habits were significant predictors of CD133 expression (Table 4). Patients who were habitual users (71\%) had notably absent CD133 expression in comparison to non-users $(P=0.003)$. The type of risk factor habit also appeared to affect CD133 expression as $69 \%$ of betel quid/areca nut users $(P=0.015)$ and $65 \%$ of chalia/ gutka/niswar users $(P=0.047)$ had tumours that did not express CD133.

Furthermore, it was seen that CD133 expression was appreciably negative in tumours with a floor of mouth involvement $(P=0.047)$. Contrarily, tumours that involved the tonsils had a $100 \%$ CD133 expression rate $(P=0.051)$.

3.4. L1CAM Expression. Positive L1CAM expression was observed as diffuse patches of dark brown membranous staining in all cases, while in some patients, it was also present on the infiltration border of the tissue (Figure 1(c)). L1CAM positivity was seen in 41 specimens, while 59 were negative for L1CAM. The positive specimens were further classified as $34(83 \%)$ mild, 5 (12\%) moderate and 2 (5\%) strong. Despite the high number of positive specimens observed L1CAM immunoexpression was not significantly affected by any of the clinicopathologic parameters or biomarkers tested (Table 4).

3.5. SOX2 Expression. Specimens positive for SOX2 expression exhibited dark brown nuclear staining (Figure 1(d)). SOX2 expression was observed in differentiated and less differentiated tissue layers alike, including the stratum basale and tumour cells resembling a basal-like phenotype. Total specimens positive for SOX2 expression were 63, while 37 did not express SOX2. The positive specimens included 32 (51\%) mild, 28 (44\%) moderate, and 3 (5\%) strong. Although SOX 2 was positive in many specimens, this did not translate into statistically significant interactions. It was seen that a large percentage of habitual smokers (71\%) had positive SOX2 expression as compared to nonsmokers $(P=0.086)$. Similarly, SOX2 expression was higher in moderately differentiated OSCC patients $(71 \%)$, but this too was borderline significant $(P=0.052)$. 
TABle 3: Patient characteristics $(n=100)$.

\begin{tabular}{|c|c|c|c|}
\hline Characteristics & No. & Characteristics & No. \\
\hline Gender & & AJCC stage & \\
\hline Male & 57 & Stage I & 19 \\
\hline Female & 43 & Stage II & 32 \\
\hline Age division & & Stage III & 23 \\
\hline$<40$ years & 18 & Stage IV & 26 \\
\hline 40 and $>40$ years & 82 & Tonsil & \\
\hline Habits & & Yes & 2 \\
\hline Yes & 79 & No & 98 \\
\hline No & 21 & Skin involvement & \\
\hline Habit pattern & & Yes & 3 \\
\hline Single & 37 & No & 97 \\
\hline Multiple & 42 & Palate & \\
\hline Non-users & 21 & Yes & 8 \\
\hline Tobacco/smoking & & No & 92 \\
\hline Yes & 35 & Mandible & \\
\hline No & 65 & Yes & 26 \\
\hline Paan/supari & & No & 74 \\
\hline Yes & 61 & Retromandibular & \\
\hline No & 39 & Yes & 16 \\
\hline Chalia/gutka/niswar & & No & 84 \\
\hline Yes & 31 & Floor of mouth & \\
\hline No & 69 & Yes & 7 \\
\hline Primary tumour site & & No & 93 \\
\hline Cheek & 63 & Surgical margins & \\
\hline Tongue & 37 & Clear & 62 \\
\hline Histological differentiation & & Near & 27 \\
\hline WDSSC & 37 & Involved & 11 \\
\hline MDSCC & 59 & Radiotherapy & \\
\hline PDSCC & 4 & Yes & 65 \\
\hline Size of primary tumour (T) & & No & 35 \\
\hline $\mathrm{T} 1$ & 21 & Overall survival & \\
\hline $\mathrm{T} 2$ & 47 & Alive & 44 \\
\hline T3 & 15 & Dead & 56 \\
\hline $\mathrm{T} 4$ & 17 & Recurrence & \\
\hline Lymph node metastasis (N) & & Yes & 74 \\
\hline No & 77 & No & 26 \\
\hline N1 & 13 & SOX-2 & \\
\hline N2 & 10 & Positive & 63 \\
\hline CD44 & & Negative & 37 \\
\hline High & 33 & SOX-2 staining intensity & \\
\hline Low & 67 & Mild & 32 \\
\hline CD133 & & Moderate & 28 \\
\hline Positive & 23 & Strong & 3 \\
\hline Negative & 77 & $L 1 C A M$ & \\
\hline CD133 staining intensity & & Positive & 41 \\
\hline Mild & 15 & Negative & 59 \\
\hline Moderate & 6 & L1CAM staining intensity & \\
\hline \multirow[t]{3}{*}{ Strong } & 2 & Mild & 34 \\
\hline & & Moderate & 5 \\
\hline & & Strong & 2 \\
\hline
\end{tabular}

3.6. Overall Survival (OS). The survival rate in our patients at minimum 60 months follow-up was $44 \%$. In Kaplan-Meier OS analysis, the median number of months for our patient cohort was 64 . The median OS was higher in males versus females and in patients $<40$ years versus $\geq 40$ years old; however, these differences were not significant. Similarly, the use of risk factors and primary tumour site was not significantly associated with survival. However, patients with subinvolvement of the tonsils had a significantly lower OS ( $P<0.001,9$ vs. 100 months) than patients with no tonsils involved. Moreover, patients with positive neck pathology had a much shorter survival as compared to patients with no lymph node involvement ( $P=0.001,31$ vs. 155 months). Equally, the involvement of multiple lymph nodes instead of single also contributed to a starkly lower OS ( $P=0.001,59$ vs. 12 months). Likewise, stage N2 patients had the lowest survival at 12 months, as compared to N0 (149 months) and N1 (31 months) stages $(P<0.001)$.

The status of surgical margins was also a key predictor of OS as those with clear margins survived the longest at 149 months, and patients with involved margins had the worst median survival of only 13 months $(P=0.004)$. The AJCC stage of patients was also a major prognostic indicator, as the survival of patients was highest for stage I patients (249 months) and was seen to steadily decrease with increasing AJCC stage until reaching worse survival for stage IV (14 months; $P=0.002$ ). In patients that received radiotherapy treatment, it was observed to significantly improve OS $(P=0.026)$. Regarding biomarkers, patients with high CD44 expression had a significantly lower median OS at 64 months compared to 106 months for patients with low CD44 expression $(P=0.047$; Figure 2$)$. Complete overall survival statistics are given in Table 5.

In Cox regression univariate analysis, the following factors were associated with a higher risk of death: tonsil involvement $(P=0.004, \mathrm{HR}=8.99)$, involved primary margins $(P=0.003, \mathrm{HR}=3.09)$, T4 tumour size $(P=0.027$, $\mathrm{HR}=2.8)$, N2 stage $(P<0.001, \mathrm{HR}=4.15)$, AJCC stage IV $(P=0.002, \quad H R=4.26), \quad$ and radiotherapy received $(P=0.029, \mathrm{HR}=1.95)$. Although CD44 was a significant predictor on Kaplan-Meier analysis, borderline significance was observed for CD44 expression in Cox regression analysis with $P=0.051$ and $H R=1.71$. All other factors tested in univariate analysis are summarized in Table 6.

3.7. Disease-Free Survival (DFS). The rate of recurrence observed in our OSCC patients at minimum 60 months follow-up was $74 \%$. In Kaplan-Meier DFS analysis, the median months for recurrence were 52.5. Factors that were significant predictors of worse OS were also seen to predict worse DFS such as: tonsil involvement $(P=0.001)$, neck pathology $(P=0.018)$, involved primary margins $(P=0.008)$, N2 stage $(P=0.024)$, and AJCC stage IV $(P=0.03)$. Additionally, cheek as primary tumour site $(P=0.045)$ and skin involvement $(P=0.031)$ were also seen to cause significantly lower median DFS months (Figure 3). For complete disease-free survival statistics, see Table 5.

In Cox regression univariate analysis, the following factors were associated with increased risk of recurrence: primary tumour site $(P=0.049, \mathrm{HR}=1.66)$, tonsil involvement $(P=0.007, \quad H R=7.69)$, skin involvement $(P=0.045, \mathrm{HR}=3.32)$, primary margins being involved $(P=0.004, \quad \mathrm{HR}=2.756), \quad \mathrm{T} 4$ tumour size $(P=0.034$, $\mathrm{HR}=2.28), \mathrm{N} 2$ stage $(P=0.025, \mathrm{HR}=2.36)$, and AJCC stage IV $(P=0.009, H R=2.64)$. Table 6 lists all factors tested for univariate DFS survival. 


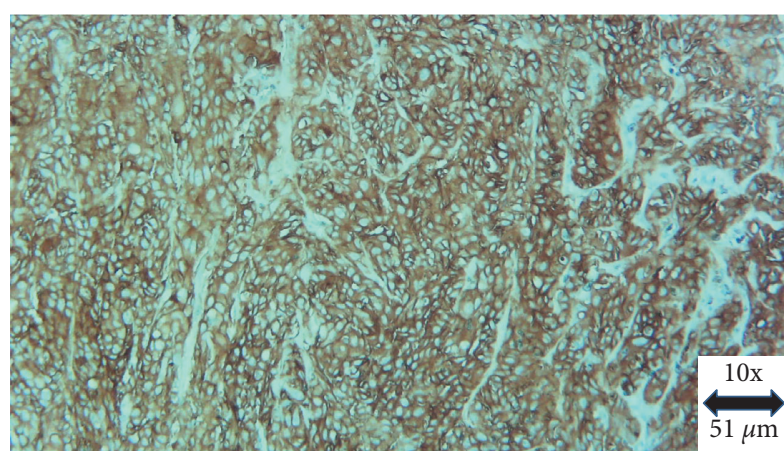

(a)

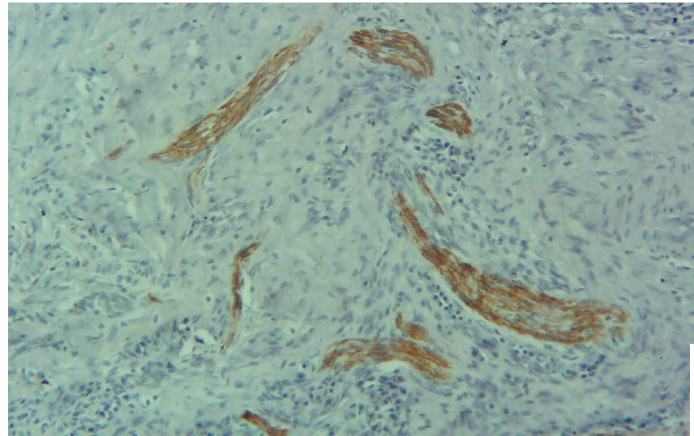

(c)

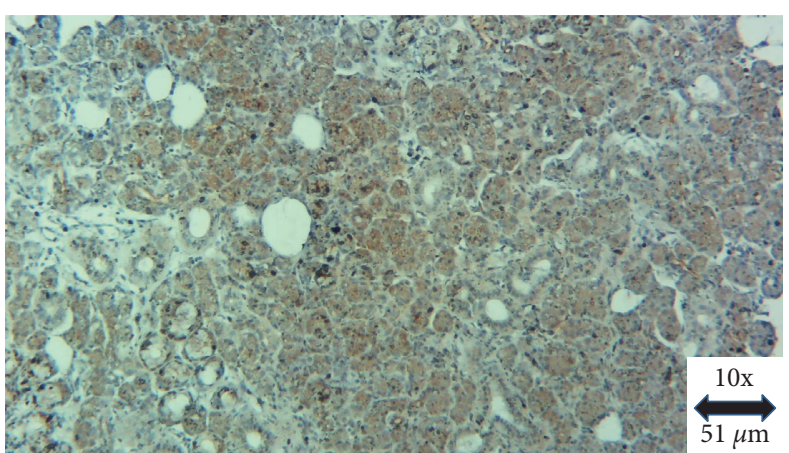

(b)

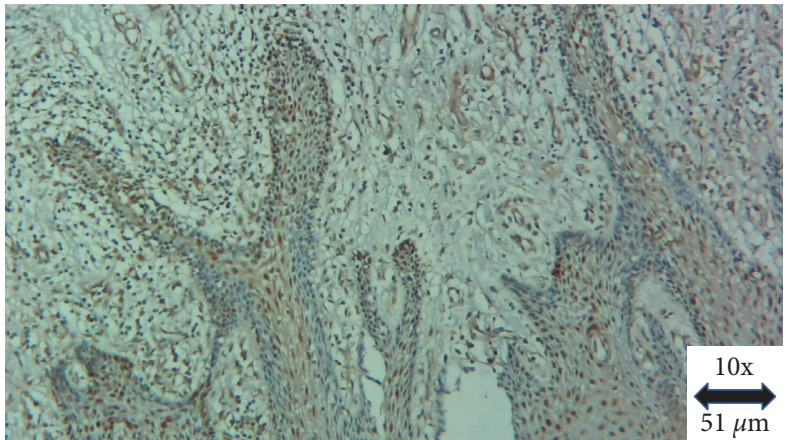

(d)

FIGURE 1: Photomicrograph of (a) CD44 cell membranous positivity, (b) CD133 cell membranous and cytoplasmic positivity, (c) L1CAM cell membranous positivity, and (d) SOX2 nuclear positivity in OSCC (magnification $\times 10$ ).

TABLE 4: Correlations of antibody expression and patient characteristics.

\begin{tabular}{|c|c|c|c|c|c|c|c|c|c|c|c|c|c|}
\hline \multirow{2}{*}{ Clinicopathologic parameters } & \multirow{2}{*}{ Total cases } & \multicolumn{3}{|c|}{ CD133 } & \multicolumn{3}{|c|}{ CD44 } & \multicolumn{3}{|c|}{ L1CAM } & \multicolumn{3}{|c|}{ SOX2 } \\
\hline & & - ve & +ve & $P$ & Low & High & $P$ & -ve & $+\mathrm{ve}$ & $P$ & $-\mathrm{ve}$ & +ve & $P$ \\
\hline \multicolumn{14}{|l|}{ Age } \\
\hline$<40$ years & 18 & 11 & 7 & 0.077 & 9 & 9 & 0.090 & 12 & 6 & 0.465 & 6 & 12 & 0.722 \\
\hline$\geq 40$ years & 82 & 66 & 16 & & 58 & 24 & & 47 & 35 & & 31 & 51 & \\
\hline \multicolumn{14}{|l|}{ Gender } \\
\hline Male & 57 & 41 & 16 & 0.165 & 38 & 19 & 0.935 & 30 & 27 & 0.136 & 19 & 38 & 0.382 \\
\hline Female & 43 & 36 & 7 & & 29 & 14 & & 29 & 14 & & 18 & 25 & \\
\hline \multicolumn{14}{|l|}{ Habits } \\
\hline Yes & 79 & 56 & 23 & $0.003^{*}$ & 54 & 25 & 0.576 & 46 & 33 & 0.761 & 30 & 49 & 0.695 \\
\hline No & 21 & 21 & 0 & & 13 & 8 & & 13 & 8 & & 7 & 14 & \\
\hline \multicolumn{14}{|l|}{ Betel quid/areca nut } \\
\hline Yes & 61 & 42 & 19 & $0.015^{*}$ & 43 & 18 & 0.353 & 37 & 24 & 0.674 & 22 & 39 & 0.809 \\
\hline No & 39 & 35 & 4 & & 24 & 15 & & 22 & 17 & & 15 & 24 & \\
\hline \multicolumn{14}{|l|}{ Smoking/tobacco use } \\
\hline Yes & 35 & 25 & 10 & 0.331 & 23 & 12 & 0.841 & 19 & 16 & 0.482 & 9 & 26 & 0.086 \\
\hline No & 65 & 52 & 13 & & 44 & 21 & & 40 & 25 & & 28 & 37 & \\
\hline \multicolumn{14}{|l|}{ Chalia/gutka/naswar } \\
\hline Yes & 31 & 20 & 11 & $0.047^{*}$ & 20 & 11 & 0.723 & 16 & 15 & 0.314 & 15 & 16 & 0.114 \\
\hline No & 69 & 57 & 12 & & 47 & 22 & & 43 & 26 & & 22 & 47 & \\
\hline \multicolumn{14}{|l|}{ Habit pattern } \\
\hline Single & 37 & 27 & 10 & 0.929 & 25 & 12 & 0.848 & 22 & 15 & 0.934 & 16 & 21 & 0.613 \\
\hline Multiple & 42 & 29 & 13 & 0.998 & 29 & 13 & 0.663 & 24 & 18 & 0.855 & 14 & 28 & 0.460 \\
\hline Non-users & 21 & 21 & 0 & 0.998 & 13 & 8 & 0.571 & 13 & 8 & 0.718 & 7 & 14 & 1 \\
\hline \multicolumn{14}{|l|}{ Primary tumour site } \\
\hline Cheek & 63 & 48 & 15 & 0.802 & 41 & 22 & 0.594 & 36 & 27 & 0.622 & 22 & 41 & 0.574 \\
\hline Tongue & 37 & 29 & 8 & & 26 & 11 & & 23 & 14 & & 15 & 22 & \\
\hline Palate & & & & & & & & & & & & & \\
\hline
\end{tabular}


TABle 4: Continued.

\begin{tabular}{|c|c|c|c|c|c|c|c|c|c|c|c|c|c|}
\hline \multirow{2}{*}{ Clinicopathologic parameters } & \multirow{2}{*}{ Total cases } & \multicolumn{3}{|c|}{ CD133 } & \multicolumn{3}{|c|}{ CD44 } & \multicolumn{3}{|c|}{ L1CAM } & \multicolumn{3}{|c|}{ SOX2 } \\
\hline & & -ve & +ve & $P$ & Low & High & $P$ & -ve & $+\mathrm{ve}$ & $P$ & -ve & $+\mathrm{ve}$ & $P$ \\
\hline Yes & 8 & 6 & 2 & 1 & 6 & 2 & 1 & 5 & 3 & 1 & 3 & 5 & 1 \\
\hline No & 92 & 71 & 21 & & 61 & 31 & & 54 & 38 & & 34 & 58 & \\
\hline \multicolumn{14}{|l|}{ Mandible } \\
\hline Yes & 26 & 22 & 4 & 0.283 & 15 & 11 & 0.241 & 14 & 12 & 0.535 & 10 & 16 & 0.858 \\
\hline No & 74 & 55 & 19 & & 52 & 22 & & 45 & 29 & & 27 & 47 & \\
\hline \multicolumn{14}{|l|}{ Floor of the mouth } \\
\hline Yes & 7 & 3 & 4 & $0.047^{*}$ & 2 & 5 & $0.038^{*}$ & 2 & 5 & 0.119 & 3 & 4 & 0.708 \\
\hline No & 93 & 74 & 19 & & 65 & 28 & & 57 & 36 & & 34 & 59 & \\
\hline \multicolumn{14}{|l|}{ Tonsils } \\
\hline Yes & 2 & 0 & 2 & 0.051 & 0 & 2 & 0.107 & 0 & 2 & 0.166 & 1 & 1 & 1 \\
\hline No & 98 & 77 & 21 & & 67 & 31 & & 59 & 39 & & 36 & 62 & \\
\hline \multicolumn{14}{|l|}{ Skin } \\
\hline Yes & 3 & 3 & 0 & 1 & 2 & 1 & 1 & 1 & 2 & 0.566 & 0 & 3 & 0.294 \\
\hline No & 97 & 74 & 23 & & 65 & 32 & & 58 & 39 & & 37 & 60 & \\
\hline \multicolumn{14}{|l|}{ Differentiation } \\
\hline Well differentiated & 37 & 25 & 12 & 0.313 & 26 & 11 & 0.790 & 21 & 16 & 0.785 & 18 & 19 & 0.131 \\
\hline Moderately differentiated & 59 & 48 & 11 & 0.127 & 38 & 21 & 0.554 & 35 & 24 & 0.804 & 17 & 42 & 0.052 \\
\hline Poorly differentiated & 4 & 4 & 0 & 0.999 & 3 & 1 & 0.844 & 3 & 1 & 0.491 & 2 & 2 & 0.959 \\
\hline \multicolumn{14}{|l|}{ Primary margins } \\
\hline Clear & 62 & 48 & 14 & 0.938 & 42 & 20 & 0.964 & 36 & 26 & 0.476 & 23 & 39 & 0.783 \\
\hline Near & 27 & 21 & 6 & 0.970 & 18 & 9 & 0.921 & 18 & 9 & 0.446 & 9 & 18 & 0.734 \\
\hline Involved & 11 & 8 & 3 & 0.735 & 7 & 4 & 0.790 & 5 & 6 & 0.440 & 5 & 6 & 0.600 \\
\hline \multicolumn{14}{|l|}{ T classification } \\
\hline $\mathrm{T} 1$ & 21 & 17 & 4 & 0.461 & 18 & 3 & 0.058 & 14 & 7 & 0.636 & 8 & 13 & 0.849 \\
\hline $\mathrm{T} 2$ & 47 & 33 & 14 & 0.358 & 32 & 15 & 0.138 & 27 & 20 & 0.474 & 16 & 31 & 0.747 \\
\hline T3 & 15 & 12 & 3 & 0.943 & 6 & 9 & $0.007^{*}$ & 7 & 8 & 0.234 & 7 & 8 & 0.608 \\
\hline $\mathrm{T} 4$ & 17 & 15 & 2 & 0.544 & 11 & 6 & 0.140 & 11 & 6 & 0.899 & 6 & 11 & 0.859 \\
\hline \multicolumn{14}{|l|}{$N$ classification } \\
\hline No & 77 & 60 & 17 & 0.856 & 54 & 23 & 0.413 & 46 & 31 & 0.921 & 27 & 50 & 0.732 \\
\hline N1 & 13 & 10 & 3 & 0.936 & 8 & 5 & 0.538 & 7 & 6 & 0.690 & 6 & 7 & 0.445 \\
\hline N2 & 10 & 7 & 3 & 0.577 & 5 & 5 & 0.209 & 6 & 4 & 0.987 & 4 & 6 & 0.759 \\
\hline \multicolumn{14}{|l|}{ AJCC clinical stage } \\
\hline I & 19 & 15 & 4 & 0.692 & 16 & 3 & 0.157 & 13 & 6 & 0.569 & 8 & 11 & 0.768 \\
\hline II & 32 & 23 & 9 & 0.576 & 23 & 9 & 0.321 & 17 & 15 & 0.286 & 10 & 22 & 0.434 \\
\hline III & 23 & 17 & 6 & 0.703 & 12 & 11 & $0.036^{*}$ & 12 & 11 & 0.288 & 10 & 13 & 0.929 \\
\hline IV & 26 & 22 & 4 & 0.624 & 16 & 10 & 0.107 & 17 & 9 & 0.831 & 9 & 17 & 0.609 \\
\hline \multicolumn{14}{|l|}{ Radiotherapy } \\
\hline Yes & 65 & 49 & 16 & 0.601 & 41 & 24 & 0.256 & 41 & 24 & 0.259 & 22 & 43 & 0.373 \\
\hline No & 25 & 28 & 7 & & 26 & 9 & & 18 & 11 & & 15 & 20 & \\
\hline CD133 & & & & & & & & & & & & & \\
\hline Positive & 23 & - & - & - & 15 & 8 & 0.836 & 10 & 13 & 0.850 & 9 & 14 & 0.809 \\
\hline Negative & 77 & - & - & & 52 & 25 & & 49 & 28 & & 28 & 49 & \\
\hline CD44 & & & & & & & & & & & & & \\
\hline High & 33 & 25 & 8 & 0.836 & - & - & - & 20 & 13 & 0.819 & 14 & 19 & 0.43 \\
\hline Low & 67 & 52 & 15 & & - & - & & 39 & 28 & & 23 & 44 & \\
\hline L1CAM & & & & & & & & & & & & & \\
\hline Positive & 41 & 28 & 13 & 0.085 & 28 & 13 & 0.819 & - & - & - & 17 & 24 & 0.441 \\
\hline Negative & 59 & 49 & 10 & & 39 & 20 & & - & - & & 20 & 39 & \\
\hline SOX 2 & & & & & & & & & & & & & \\
\hline Positive & 63 & 49 & 14 & 0.809 & 44 & 19 & 0.43 & 39 & 24 & 0.441 & - & - & - \\
\hline Negative & 37 & 28 & 9 & & 23 & 14 & & 20 & 17 & & - & - & \\
\hline
\end{tabular}

${ }^{*} P<0.05$ taken as significant.

\section{Discussion}

Oral cancer is a heterogeneous disease, arising from the dysfunction of several molecular pathways, resulting in severe morbidity and oftentimes mortality. The survival of OSCC patients has remained largely unchanged for the past 40 years [19]. CSCs represent a group of markers that may be used to successfully estimate prognosis and serve as targets for molecular therapy, as CSC markers are mainly expressed in the basal layers of the oral mucosal surfaces and have frequently dysregulated expression in OSCC. 

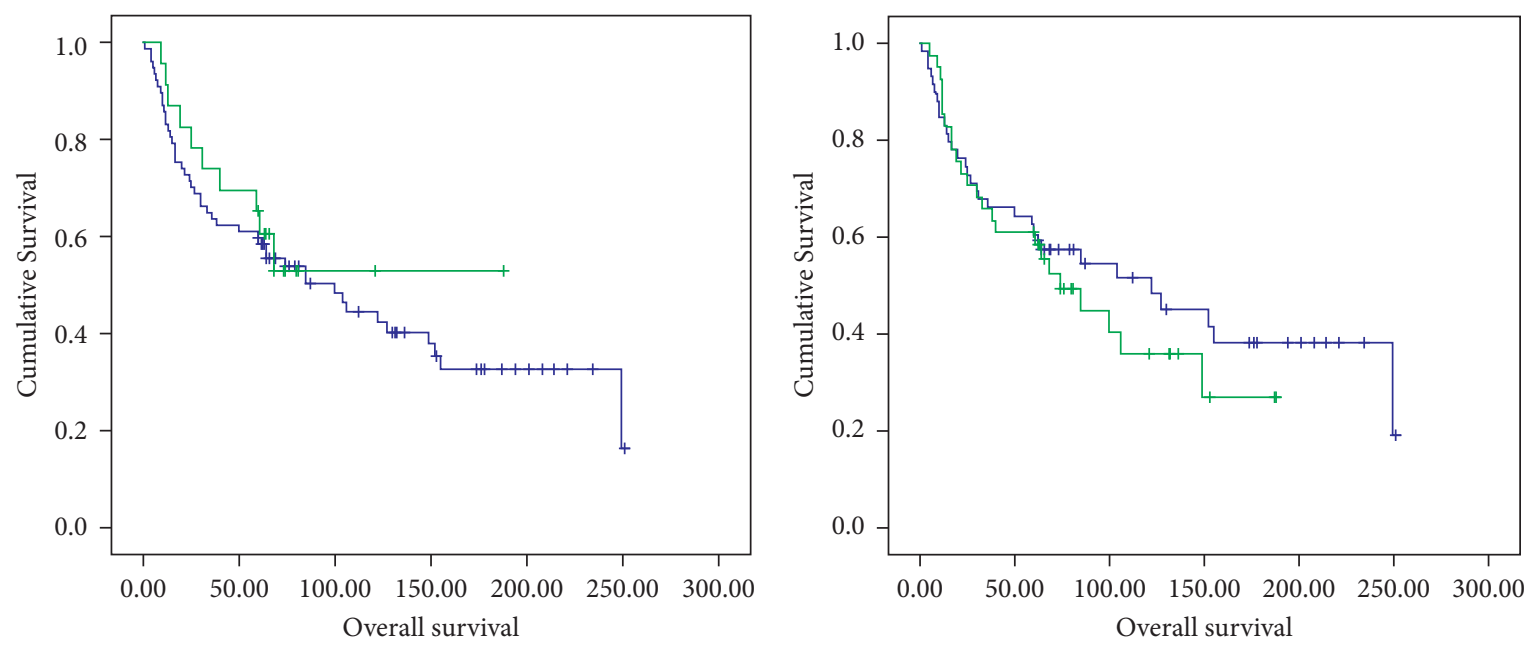

CD133 Immunohistochemistry
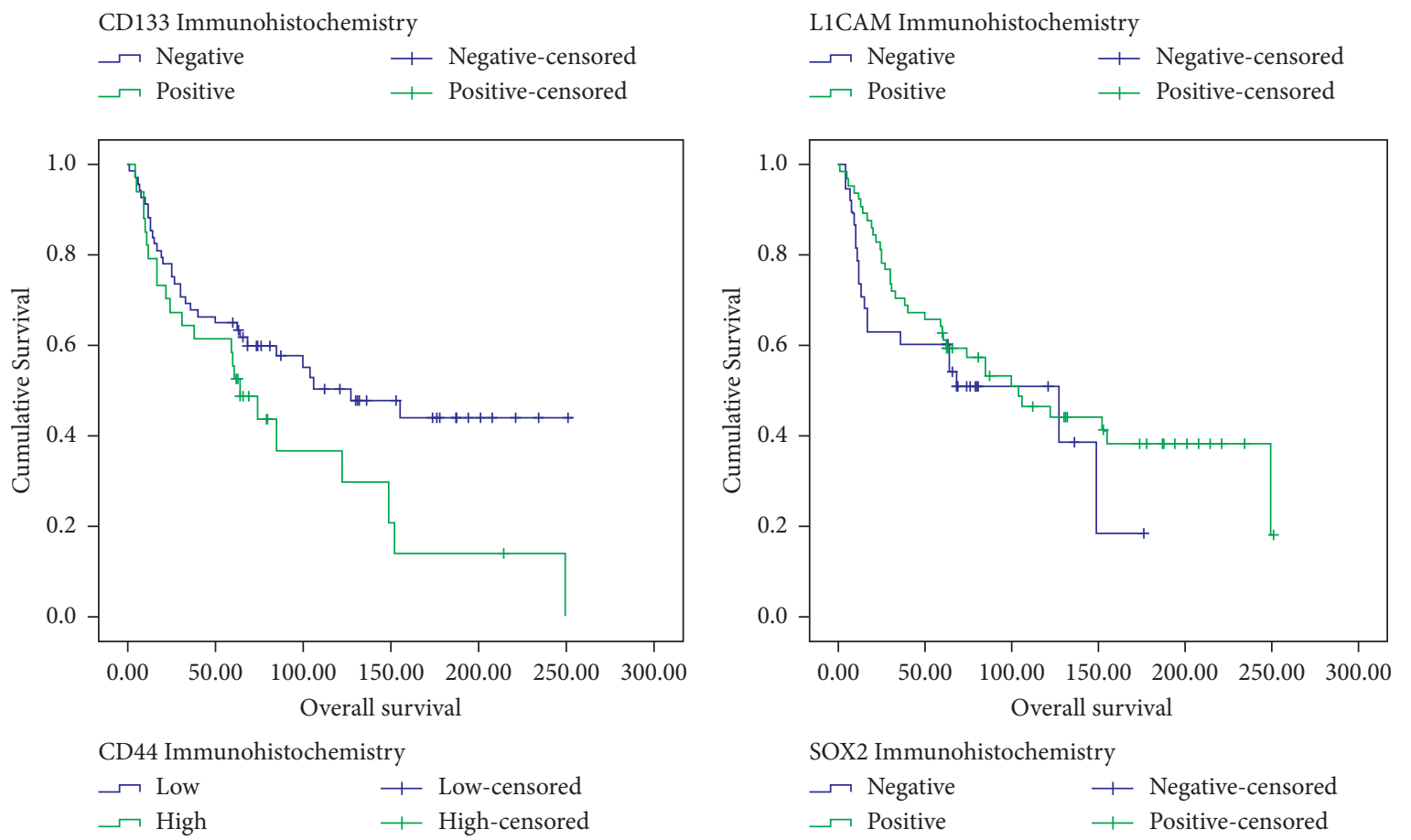

Figure 2: Kaplan-Meier curve analysis for overall survival of OSCC patients with $P=0.613$ for CD133 expression, $P=0.047$ for CD44 expression, $P=0.489$ for L1CAM expression, and $P=0.318$ for SOX2 expression.

High CD44 expression was recently observed to be an independent predictor for prognosis in a study of 44 patients by Hendawy and Esmail [7]. The authors found that CD44 was increased in patients with advanced TNM stage and that it led to reduced DFS and 3-year OS. Although we found a lesser positivity percentage (33\%) as compared to Esmail et al.'s (59\%), the negative impact on overall survival was noted in both studies. Although CD44 led to a poor prognosis, a correlation with DFS was not determined in this cohort. This is similar to the conclusions of another study that found abundant CD44 expression in stage I and II OSCC cells but no correlation with disease recurrence [20]. However, another study group determined reduced DFS for CD44 positive patients [7]. The difference in positive cases can be attributed to the dissimilar genetic makeup of the populations under study, Egyptian and Pakistani, though the same antibody clone and similar scoring criteria were applied in both studies.

It is hypothesized that CD44 affects patient survival by conferring radio- and chemoresistance in the tumours and causing relapse and metastasis. Moreover, CD44 stimulates pathways that initiate and promote tumour cell proliferation and epithelial-to-mesenchymal transition [21]. This seems to be the case in this study as participants had advanced disease and moderately differentiated carcinomas.

The exact location of CD44 staining is also thought to influence prognosis. Boxberg et al. [22] compared the expression of CD44 within the tumour core, at the invasive margin, and in lymph node metastases; the invasive margin had the highest expression of all sites (39\%) and was an independent predictor for worse survival and recurrence. 
TABLe 5: Kaplan-Meier (log-rank statistic) analysis for overall survival and disease-free survival $(n=100)$.

\begin{tabular}{|c|c|c|c|c|c|c|c|c|c|}
\hline \multirow{2}{*}{ Variable } & \multirow{2}{*}{ Total } & \multirow{2}{*}{$\begin{array}{l}\text { OS in months } \\
\text { Median }\end{array}$} & \multirow{2}{*}{$P$} & \multicolumn{2}{|c|}{$95 \% \mathrm{CI}$} & \multirow{2}{*}{$\begin{array}{l}\text { DFS in months } \\
\text { Median }\end{array}$} & \multirow{2}{*}{$P$} & \multicolumn{2}{|c|}{$95 \% \mathrm{CI}$} \\
\hline & & & & Lower & Upper & & & Lower & Upper \\
\hline \multicolumn{10}{|l|}{ Gender } \\
\hline Male & 57 & 100 & 0.597 & 37.7 & 162.3 & 51 & 0.629 & 28.1 & 73.9 \\
\hline Female & 43 & 85 & & 30.1 & 139.4 & 58 & & 35.4 & 80.6 \\
\hline \multicolumn{10}{|c|}{ Age division } \\
\hline$<40$ years & 18 & 155 & 0.93 & & & 31 & 0.376 & 0 & 80.9 \\
\hline$>40$ years & 82 & 100 & & 60.2 & 139.8 & 52 & & 35.1 & 69 \\
\hline \multicolumn{10}{|c|}{ Primary tumour site } \\
\hline Cheek & 63 & 85 & 0.287 & 46.6 & 123.4 & 44 & $0.045^{*}$ & 21.6 & 66.4 \\
\hline Tongue & 37 & 155 & & & & 58 & & 20.1 & 95.8 \\
\hline \multicolumn{10}{|l|}{ Tonsil } \\
\hline Yes & 2 & 9 & $<0.001^{*}$ & & & 5 & $0.001^{*}$ & & \\
\hline No & 98 & 100 & & 53.1 & 146.9 & 53 & & 40.8 & 65.2 \\
\hline \multicolumn{10}{|l|}{ Skin } \\
\hline Yes & 3 & 25 & 0.082 & 4.2 & 45.8 & 7 & $0.031^{*}$ & 2.2 & 11.9 \\
\hline No & 97 & 104 & & 54.6 & 153.5 & 53 & & 37.1 & 68.9 \\
\hline \multicolumn{10}{|c|}{ Neck pathology } \\
\hline Positive & 27 & 31 & $0.001^{*}$ & 0 & 102.2 & 69 & $0.018^{*}$ & 51.8 & 86.2 \\
\hline Negative & 51 & 155 & & & & 22 & & 0 & 52.5 \\
\hline ND & 22 & 64 & & 13 & 115 & 27 & & 4 & 50 \\
\hline \multicolumn{10}{|c|}{ Pathologically involved lymph nodes } \\
\hline Single & 16 & 59 & $0.001^{*}$ & 2.2 & 115.8 & 29 & 0.078 & 0 & 85.8 \\
\hline Multiple & 11 & 12 & & 6.6 & 17.4 & 6 & & 2.8 & 9.2 \\
\hline NA & 73 & 149 & & 85.4 & 212.6 & 58 & & 40.9 & 75.1 \\
\hline \multicolumn{10}{|c|}{ Primary margins } \\
\hline Clear & 62 & 149 & $0.004^{*}$ & 88.5 & 209.5 & 62 & $0.008^{*}$ & 49 & 75 \\
\hline Near & 27 & 62 & & 23.2 & 100.8 & 24 & & 0 & 76.6 \\
\hline Involved & 11 & 13 & & 9.8 & 16.2 & 7 & & 0 & 15.6 \\
\hline \multicolumn{10}{|c|}{$N$ classification } \\
\hline No & 77 & 149 & $<0.001^{*}$ & 79.2 & 218.8 & 58 & $0.024^{*}$ & 48.6 & 67.4 \\
\hline N1 & 13 & 31 & & 11.6 & 50.4 & 27 & & 5.9 & 48.1 \\
\hline N2 & 10 & 12 & & 9 & 15 & 6 & & 2.9 & 9.1 \\
\hline \multicolumn{10}{|c|}{ AJCC stage } \\
\hline I & 19 & 249 & $0.002^{*}$ & 34.7 & 463.3 & 69 & $0.03^{*}$ & 51.8 & 86.3 \\
\hline II & 32 & 149 & & 82.5 & 215.5 & 58 & & 39.7 & 76.3 \\
\hline III & 23 & 68 & & 47.5 & 88.5 & 51 & & 22.8 & 79.2 \\
\hline IV & 26 & 14 & & 0 & 30.2 & 9 & & 2 & 16 \\
\hline Radiothero & & & & & & & & & \\
\hline Yes & 65 & 68 & $0.026^{*}$ & 43.5 & 92.5 & 44 & 0.242 & 18.3 & 69.7 \\
\hline No & 35 & & & & & 62 & & 35 & 89 \\
\hline CD44 & & & & & & & & & \\
\hline High & 33 & 64 & $0.047^{*}$ & 46.8 & 81.2 & 45 & 0.24 & 11.2 & 78.8 \\
\hline Low & 67 & 106 & & 39.9 & 172.1 & 57 & & 39.3 & 74.7 \\
\hline CD133 & & & & & & & & & \\
\hline Positive & 23 & & 0.613 & & & 57 & 0.996 & 35.1 & 79 \\
\hline Negative & 77 & 100 & & 55.5 & 144.5 & 52 & & 24.5 & 79.6 \\
\hline$L 1 \stackrel{\circ}{C} A M$ & & & & & & & & & \\
\hline Positive & 41 & 74 & 0.489 & 42.7 & 105.3 & 44 & 0.266 & 5.1 & 82.9 \\
\hline Negative & 59 & 122 & & 42.4 & 201.6 & 53 & & 40.2 & 65.8 \\
\hline SOX2 & & & & & & & & & \\
\hline Positive & 63 & 100 & 0.318 & 53.7 & 146.3 & 53 & 0.483 & 39.8 & 66.2 \\
\hline Negative & 37 & 68 & & & 136.4 & 51 & & 0 & 104.6 \\
\hline
\end{tabular}

${ }^{*} P<0.05$ taken as significant.

On the other hand, Cohen et al. [23] studied a diverse population of black and Hispanic ethnicities and found that universal gross staining rather than peripheral staining was associated with poor overall survival. As they found a relatively high positivity of $62.5 \%$ in 40 specimens, it was concluded that the percentage of cells expressing CD44 was more influential on prognosis as compared to staining intensity or localization. This is also reflected in current study results as 33\% CD44 universal staining led to worse patient survival. 
TABLE 6: Cox regression univariate analysis $(n=100)$.

\begin{tabular}{|c|c|c|c|c|c|c|c|c|}
\hline \multirow{3}{*}{ Characteristic } & \multicolumn{4}{|c|}{ Overall survival } & \multicolumn{4}{|c|}{ Disease-free survival } \\
\hline & \multirow{2}{*}{$P$} & \multirow{2}{*}{$\begin{array}{c}\text { Hazard ratio } \\
\text { HR }\end{array}$} & \multicolumn{2}{|c|}{$95 \% \mathrm{CI}$} & \multirow{2}{*}{$P$} & \multirow{2}{*}{$\begin{array}{c}\text { Hazard ratio } \\
\text { HR }\end{array}$} & \multicolumn{2}{|c|}{$95 \% \mathrm{CI}$} \\
\hline & & & Lower & Upper & & & Lower & Upper \\
\hline Gender & & 100 & & & & 100 & & \\
\hline Male & & 1.0 (ref) & & & & 1.0 (ref) & & \\
\hline Female & 0.598 & 1.154 & 0.678 & 1.962 & 0.632 & 0.893 & 0.562 & 1.419 \\
\hline Age division & & 100 & & & & 100 & & \\
\hline$<40$ Years & & 1.0 (ref) & & & & 1.0 (ref) & & \\
\hline$\geq 40$ Years & 0.931 & 0.969 & 0.472 & 1.987 & 0.381 & 0.769 & 0.427 & 1.385 \\
\hline$\overline{\text { Primary tumour site }}$ & & 100 & & & & 100 & & \\
\hline Tongue & & 1.0 (ref) & & & & 1.0 (ref) & & \\
\hline Cheek & 0.291 & 0.738 & 0.419 & 1.297 & $0.049^{*}$ & 1.655 & 1.002 & 2.734 \\
\hline Tonsil & & 100 & & & & 100 & & \\
\hline No & & 1.0 (ref) & & & & 1.0 (ref) & & \\
\hline Yes & $0.004^{*}$ & 8.999 & 2.016 & 40.167 & $0.007^{*}$ & 7.691 & 1.731 & 34.18 \\
\hline Skin & & 100 & & & & 100 & & \\
\hline No & & 1.0 (ref) & & & & 1.0 (ref) & & \\
\hline Yes & 0.097 & 2.699 & 0.836 & 8.718 & $0.045^{*}$ & 3.316 & 1.029 & 10.69 \\
\hline Primary margins & & 100 & & & & 100 & & \\
\hline Clear & $0.007^{*}$ & 1.0 (ref) & & & 0.11 & 1.0 (ref) & & \\
\hline Near & $0.045^{*}$ & 1.856 & 1.014 & 3.396 & 0.109 & 1.535 & 0.909 & 2.594 \\
\hline Involved & $0.003^{*}$ & 3.091 & 1.454 & 6.572 & $0.004^{*}$ & 2.756 & 1.372 & 5.537 \\
\hline T classification & & 100 & & & & 100 & & \\
\hline $\mathrm{T} 1$ & 0.135 & 1.0 (ref) & & & 0.156 & 1.0 (ref) & & \\
\hline $\mathrm{T} 2$ & 0.215 & 1.655 & 0.746 & 3.675 & 0.39 & 1.324 & 0.698 & 2.512 \\
\hline $\mathrm{T} 3$ & 0.085 & 2.23 & 0.896 & 5.548 & 0.162 & 1.732 & 0.802 & 3.743 \\
\hline $\mathrm{T} 4$ & $0.027^{*}$ & 2.803 & 1.124 & 6.991 & $0.034^{*}$ & 2.28 & 1.066 & 4.874 \\
\hline$N$ classification & & 100 & & & & 100 & & \\
\hline No & $<0.001^{*}$ & 1.0 (ref) & & & $0.031^{*}$ & 1.0 (ref) & & \\
\hline N1 & $0.004^{*}$ & 2.888 & 1.415 & 5.89 & 0.082 & 1.833 & 0.925 & 3.635 \\
\hline $\mathrm{N} 2$ & $<0.001^{*}$ & 4.148 & 1.89 & 9.103 & $0.025^{*}$ & 2.364 & 1.114 & 5.018 \\
\hline AJCC stage & & 100 & & & & 100 & & \\
\hline I & $0.004^{*}$ & 1.0 (ref) & & & $0.038^{*}$ & 1.0 (ref) & & \\
\hline II & 0.345 & 1.579 & 0.612 & 4.075 & 0.436 & 1.329 & 0.65 & 2.717 \\
\hline III & $0.033^{*}$ & 2.789 & 1.088 & 7.129 & 0.143 & 1.755 & 0.827 & 3.726 \\
\hline IV & $0.002^{*}$ & 4.259 & 1.681 & 10.792 & $0.009^{*}$ & 2.644 & 1.269 & 5.512 \\
\hline Radiotherapy & & 100 & & & & 100 & & \\
\hline Yes & & 1.0 (ref) & & & & 1.0 (ref) & & \\
\hline No & $0.029^{*}$ & 1.950 & 1.071 & 3.552 & 0.247 & 0.749 & 0.459 & 1.222 \\
\hline CD44 & & 100 & & & & 100 & & \\
\hline Low & & 1.0 (ref) & & & & 1.0 (ref) & & \\
\hline High & 0.051 & 1.71 & 0.999 & 2.927 & 0.246 & 1.346 & 0.815 & 2.223 \\
\hline CD133 & & 100 & & & & 100 & & \\
\hline Negative & & 1.0 (ref) & & & & 1.0 (ref) & & \\
\hline Positive & 0.615 & 0.837 & 0.418 & 1.676 & 0.996 & 0.998 & 0.568 & 1.756 \\
\hline L1CAM & & 100 & & & & 100 & & \\
\hline Negative & & 1.0 (ref) & & & & 1.0 (ref) & & \\
\hline Positive & 0.491 & 1.208 & 0.706 & 2.066 & 0.272 & 1.304 & 0.812 & 2.092 \\
\hline SOX2 & & 100 & & & & 100 & & \\
\hline Negative & & 1.0 (ref) & & & & 1.0 (ref) & & \\
\hline Positive & 0.321 & 1.247 & 0.668 & 2.325 & 0.265 & 1.364 & 0.79 & 2.353 \\
\hline
\end{tabular}

${ }^{*} P<0.05$ taken as significant.

An interesting observation in our data set was a significant number of the floor of the mouth tumours having high CD44 positivity. This was also noted by Krump and Ehrmann [24] who found a total of $62 \%$ positive specimens and significantly increased CD44 expression in the floor of the mouth tumours as compared to the tongue. This leads to the conclusion that the prognostic value of CD44 depends not only on the total expression in tumour but also on tumour location and maybe even on subcellular location.

Moreover, Hendawy [7] also found markedly higher CD44 expression in tumours of bigger size, overall higher TNM stage, lymphovascular invasion, and metastasis. Although similar correlations of CD44 with advanced $\mathrm{T}$ and AJCC stage were seen in this study, no effect of CD44 immunoexpression was observed on nodal involvement. 

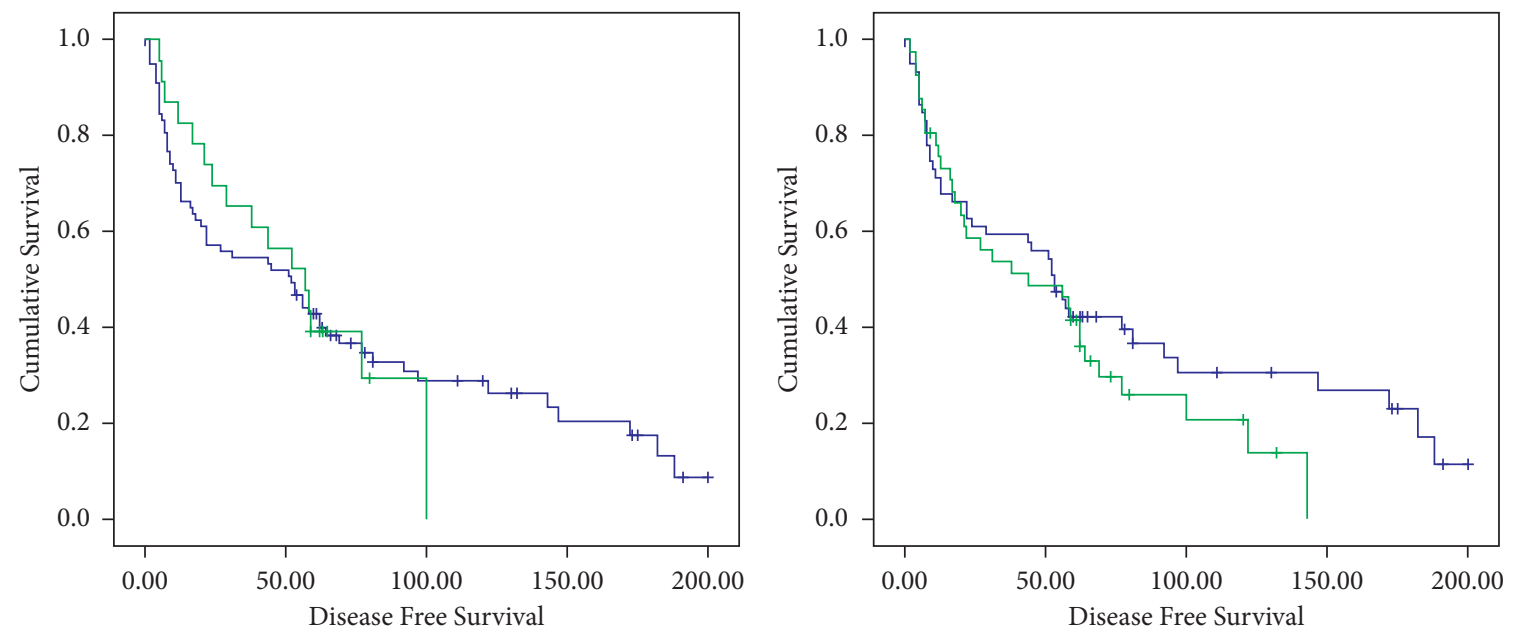

CD133 Immunohistochemistry
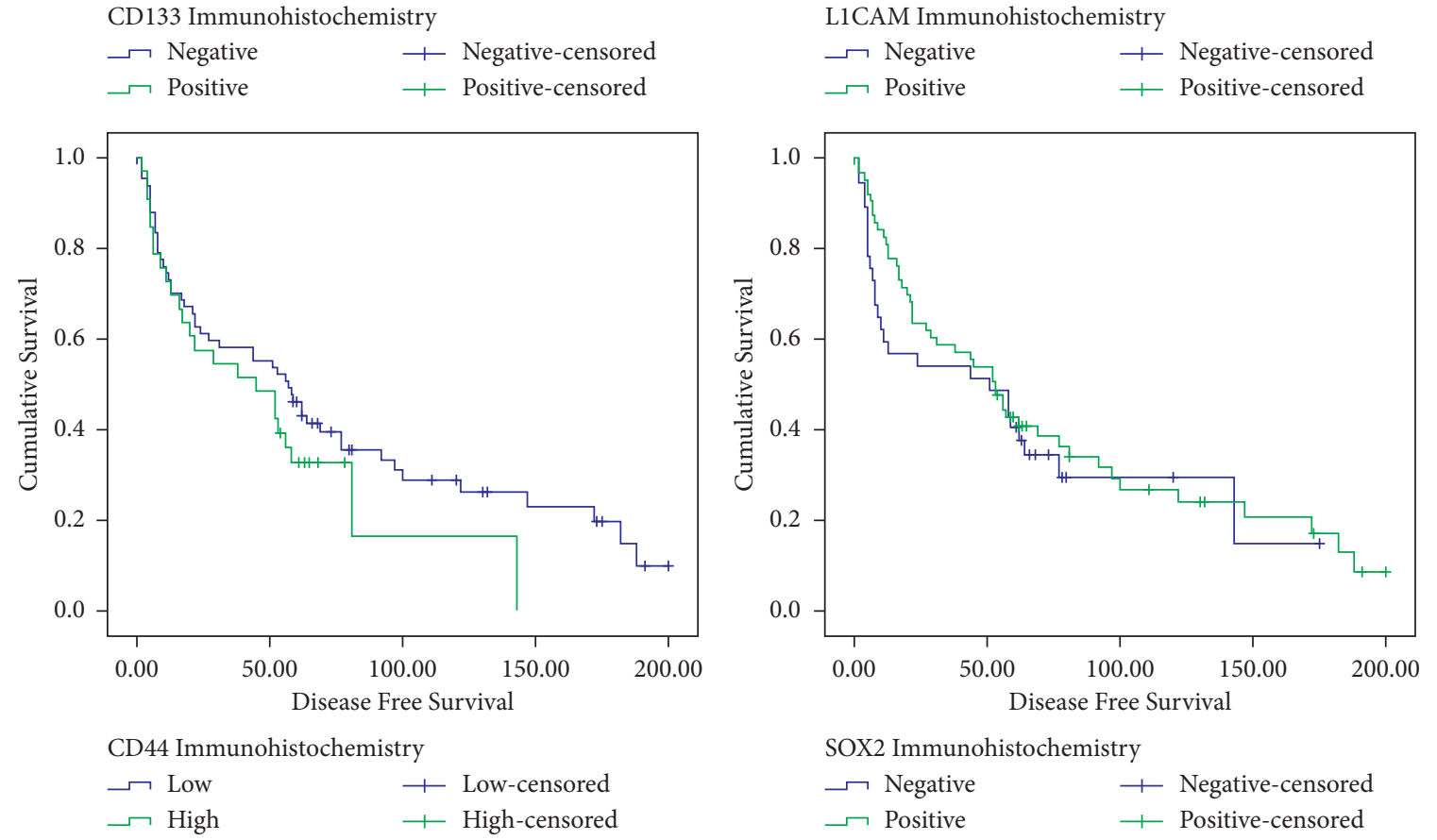

Figure 3: Kaplan-Meier curve analysis for disease-free survival of OSCC patients with $P=0.996$ for CD133 expression, $P=0.24$ for CD44 expression, $P=0.266$ for L1CAM expression, and $P=0.483$ for SOX2 expression.

Furthermore, no patients included in this study had metastasis, due to which comparisons cannot be drawn.

In the case of CD133, 23\% positivity was observed with the majority $(15 / 23)$ being mild positive. Other groups investigating CD133 expression have reported wide-ranging figures including 5.8\% [17], 68\% [25], and even $100 \%$ positivity [26].

There were unremarkable survival differences among the CD133+ and CD133- patient groups. Similarly, several other groups investigating CD133 expression in the oral cavity found no associations either patient characteristics or survival $[17,25,26]$. On the other hand, the progression of oral potentially malignant disorders to squamous cell carcinoma has been linked to high CD133 expression in premalignant specimens $[27,28]$. It is hypothesized that CD133 may play a role in initiating malignancy in early stages and cease to be a key regulator once carcinoma has fully developed. Since the patients of this study all had fully developed and advanced OSCC, the role of CD133 was not prominently observed.

Another observation was that patients who were habitual chewers of oral cancer risk products such as areca nut, betel quid, smoking and smokeless tobacco, and so on were more prone to having CD133- tumours. As per the author's knowledge, this has not been reported before. This may be explained by the fact that patients with chewing habits develop usually potentially malignant conditions, and some authors have found that CD133-cell populations may be more tumourigenic than CD133+ cells [29], ultimately causing the patients to undergo malignant transformation. In our team's experience, patients continued their addictive risk factor habits even during and immediately after treatment, despite regular counselling. Due to these prevalent habits, the genetic makeup of OSCC in Pakistani patients is 
bound to differ from Western literature. Although other CSC markers were investigated in association with betel chewing in the population of Taiwan and Sri Lanka, but no significant effect of risk factor habits on markers expression was seen [30, 31].

Furthermore, 63\% positivity was observed for SOX2 in the present study, while previous reports have varied widely with as low as 7\% [18] and as high as $100 \%$ [13] reported SOX2 positivity.

High SOX2 protein expression was observed in inpatients with moderately differentiated OSCC, but this was borderline significant $(P=0.052)$. These may be etiologic findings since a meta-analysis of SOX2 expression in head and neck cancer found that high immunoexpression leads to worse five-year survival [32]. Contrarily, other authors have suggested smaller tumour size and improved DFS for SOX2 expressing tumours [12].

The differences in findings may be due to the highly variable thresholds for positivity that have been used and also the classifications of positive staining into diffuse and peripheral patterns, with the diffuse pattern exhibiting lymph node metastasis and poorer survival [13]. Furthermore, a study utilizing a rabbit polyclonal antibody and similar staining criteria as the present study found that SOX2 was involved more in the early tumourigenesis events rather than the progression of developed OSCCs [18]. They detected SOX2 overexpression as an independent predictor of malignant transformation for oral leucoplakia, while SOX2 expression in OSCC was associated with early Tand $\mathrm{N}$ stages and better survival. Since our cohort did not include premalignant conditions, these findings were not reproduced.

Regarding L1CAM, as per our understanding, this is the first time that L1CAM immunoexpression was correlated with survival in OSCC. Since $41 \%$ of tumours were positive for L1CAM, it cannot be ruled out as a CSC marker for OSCC. Although previous findings indicate that increased L1CAM expression leads to poor histologic differentiation [15], these were not replicated in the present study cohort as L1CAM positivity was roughly inversely proportional to histological differentiation. However, the sample size in the cited study was only 25 OSCCs, while we studied 100 OSCCs and found no such association. Moreover, the percentage positivity of L1CAM and scoring criteria used was also not fully elaborated in the above-cited study.

In this group, the rate of survival was significantly lower in patients who suffered recurrence as compared to those who did not: patients with recurrence had 38 times higher risk of death. It was reported by Camisasca et al. [33] that the 5 -year survival rate was 3 times lower in patients with recurrence than those without. Several other reports have assessed the effect of patient clinicopathologic factors on survival, and in line with the majority of studies, we found conventional and established prognostic indicators, such as involved lymph nodes, higher AJCC and TNM stages, and involved surgical margins, were all significantly associated with OS and DFS in this patient cohort [34].

Over the past decade, hundreds of biomarkers for OSCC have been studied in numerous studies, but none of them has been adopted into clinical practice. This is often due to small sample sizes, inadequate validation of the marker using multiple techniques, and dearth of prospective studies.
Nevertheless, the present study adds unique insights to our understanding of oral cancer using a panel of CSC markers on the same well-characterized cohort from a resourceconstrained high-risk population. The present work sheds light on a population that is at high risk for oral cancer and ironically is much less studied due to limited scientific resources. Cancer stem cell markers help identify a subset of the tumour population that is responsible for the bulk of tumour-related characteristics and resists conventional treatment. Once this subpopulation is identified, in the next step, it can be targeted so that this self-renewal of the tumour can be halted, and complete remission can be achieved.

\section{Conclusion}

The present study found that high CD44 protein expression correlated with adverse overall survival of OSCC patients. Moreover, increased CD44 immunoexpression was more common in patients with AJCC stage III and T3 tumours. On the other hand, CD133 was significantly lower in patients with chewing habits but did not ultimately change the prognosis. SOX2 and L1CAM were impartial for OS and DFS, while tonsils, nodal involvement, and AJCC stage were independent predictors of poor OS and DFS.

\section{Data Availability}

The patient data used to support the findings of this study are included within the article.

\section{Conflicts of Interest}

The authors declare that there are no conflicts of interest.

\section{Authors' Contributions}

YA, SMAA, MSA, and HAK conceptualized the study. YA, HAF, and MSA contributed to data curation. YA, SMAA, and HAK had done the formal analysis. SMAA and MSA did the funding acquisition. YA, HAF, and RI performed the investigation. SMAA and RI contributed to the visualization. $Y A$ and SMAA prepared the original draft of the manuscript. YA, SMAA, HAF, HAK, RI, and MSA reviewed and edited the manuscript.

\section{Acknowledgments}

This work was supported by a grant awarded to Dr. S. M. Adnan Ali by Higher Education Commission (Grant ID: 9516/Sindh/NRPU/R\&D/HEC/2017).

\section{References}

[1] M. A. Qureshi, S. A. Syed, and S. Sharafat, "Lip and oral cavity cancers (C00-C06) from a mega city of Pakistan: ten-year data from the dow cancer registry," Journal of Taibah University Medical Sciences, vol. 16, no. 4, pp. 624-627, 2021.

[2] H. Sung, J. Ferlay, R. L. Siegel et al., "Global cancer statistics 2020: GLOBOCAN estimates of incidence and mortality worldwide for 36 cancers in 185 countries," CA: A Cancer Journal for Clinicians, vol. 71, no. 3, pp. 209-249, 2021. 
[3] S. M. Adnan Ali, M. S. Awan, S. Atif, N. Ali, and Y. Mirza, "Correlation of human papillomavirus infection and clinical parameters with five-year survival in oral squamous cell carcinoma," Journal of Laryngology \& Otology, vol. 132, no. 7, pp. 628-635, 2018.

[4] B. T. Tan, C. Y. Park, L. E. Ailles, and I. L. Weissman, "The cancer stem cell hypothesis: a work in progress," Laboratory Investigation, vol. 86, no. 12, pp. 1203-1207, 2006.

[5] S. S. Yu and N. Cirillo, "The molecular markers of cancer stem cells in head and neck tumors," Journal of Cellular Physiology, vol. 235, no. 1, pp. 65-73, 2020.

[6] Y. Yan, X. Zuo, and D. Wei, "Concise review: emerging role of CD44 in cancer stem cells: a promising biomarker and therapeutic target," Stem Cells Translational Medicine, vol. 4, no. 9, pp. 1033-1043, 2015.

[7] H. Hendawy, A. D. Esmail, A. M. N. Zahani, H. Elmahdi, and A. Ibrahiem, "Clinicopathological correlation of stem cell markers expression in oral squamous cell carcinoma; relation to patients' outcome," Journal of Immunoassay and Immunochemistry, vol. 42, no. 6, pp. 571-595, 2021.

[8] N. Saghravanian, K. Anvari, N. Ghazi, B. Memar, M. Shahsavari, and M. A. Aghaee, "Expression of p63 and CD44 in oral squamous cell carcinoma and correlation with clinicopathological parameters," Archives of Oral Biology, vol. 82, pp. 160-165, 2017.

[9] C.-C. Yu, F.-W. Hu, C.-H. Yu, and M.-Y. Chou, "Targeting CD133 in the enhancement of chemosensitivity in oral squamous cell carcinoma-derived side population cancer stem cells," Head \& Neck, vol. 38, no. S1, pp. E231-E238, 2016.

[10] D. Mizrak, M. Brittan, and M. Alison, "CD133: molecule of the moment," The Journal of Pathology, vol. 214, no. 1, pp. 3-9, 2008.

[11] S. Zhang, X. Xiong, and Y. Sun, "Functional characterization of SOX2 as an anticancer target," Signal Transduction and Targeted Therapy, vol. 5, no. 1, pp. 1-17, 2020.

[12] T.-Y. Fu, I.-C. Hsieh, J.-T. Cheng et al., "Association of OCT4, SOX2, and NANOG expression with oral squamous cell carcinoma progression," Journal of Oral Pathology \& Medicine, vol. 45, no. 2, pp. 89-95, 2016.

[13] Y. Michifuri, Y. Hirohashi, T. Torigoe et al., "High expression of ALDH1 and SOX2 diffuse staining pattern of oral squamous cell carcinomas correlates to lymph node metastasis," Pathology International, vol. 62, no. 10, pp. 684-689, 2012.

[14] M. Giordano and U. Cavallaro, "Different shades of L1CAM in the pathophysiology of cancer stem cells," Journal of Clinical Medicine, vol. 9, no. 5, p. 1502, 2020.

[15] S.-C. Hung, I.-H. Wu, S.-S. Hsue et al., "Targeting 11 cell adhesion molecule using lentivirus-mediated short hairpin RNA interference reverses aggressiveness of oral squamous cell carcinoma," Molecular Pharmaceutics, vol. 7, no. 6, pp. 2312-2323, 2010.

[16] S. Keren, Z. Shoude, Z. Lu, and Y. Beibei, "Role of EGFR as a prognostic factor for survival in head and neck cancer: a metaanalysis," Tumor Biology, vol. 35, no. 3, pp. 2285-2295, 2014.

[17] F. P. P. de Moraes, S. V. Lourenço, R. C. F. Ianez et al., "Expression of stem cell markers in oral cavity and oropharynx squamous cell carcinoma," Oral surgery, oral medicine, oral pathology and oral radiology, vol. 123, no. 1, pp. 113-122, 2017.

[18] J. C. de Vicente, P. Donate-Pérez del Molino, J. P. Rodrigo et al., "SOX2 expression is an independent predictor of oral cancer progression," Journal of Clinical Medicine, vol. 8, no. 10, p. 1744, 2019.
[19] J. d. S. Moro, Oral and Oropharyngeal Cancer: Epidemiology and Survival Analysis, Einstein, Sao Paulo, Brazil, 2018.

[20] T. Tamatani, N. Takamaru, G. Ohe, K. Akita, T. Nakagawa, and Y. Miyamoto, "Expression of CD44, CD44v9, ABCG2, CD24, Bmi-1 and ALDH1 in stage I and II oral squamous cell carcinoma and their association with clinicopathological factors," Oncology Letters, vol. 16, no. 1, pp. 1133-1140, 2018.

[21] H. Xu, M. Niu, X. Yuan, K. Wu, and A. Liu, "CD44 as a tumor biomarker and therapeutic target," Experimental Hematology \& Oncology, vol. 9, no. 1, pp. 36-14, 2020.

[22] M. Boxberg, C. Götz, S. Haidari et al., "Immunohistochemical expression of CD44 in oral squamous cell carcinoma in relation to histomorphological parameters and clinicopathological factors," Histopathology, vol. 73, no. 4, pp. 559-572, 2018.

[23] E. R. Cohen, I. M. Reis, C. Gomez et al., "Immunohistochemistry analysis of CD44, EGFR, and p16 in oral cavity and oropharyngeal squamous cell carcinoma," OtolaryngologyHead and Neck Surgery, vol. 157, no. 2, pp. 239-251, 2017.

[24] M. Krump and J. Ehrmann, "Differences in CD44s expression in HNSCC tumours of different areas within the oral cavity," Biomedical Papers, vol. 157, no. 4, pp. 280-283, 2013.

[25] A. Singh, A. N. Srivastava, S. Akhtar, M. H. Siddiqui, P. Singh, and V. Kumar, "Correlation of CD133 and Oct-4 expression with clinicopathological and demographic parameters in oral squamous cell carcinoma patients," National Journal of Maxillofacial Surgery, vol. 9, pp. 8-13, 2018.

[26] E. C. M. Luna, T. M. M. Bezerra, P. G. d. Barros Silva et al., "CD133 role in oral carcinogenesis," Asian Pacific Journal of Cancer Prevention, vol. 21, no. 9, pp. 2501-2506, 2020.

[27] L. Sun, J. Feng, L. Ma, W. Liu, and Z. Zhou, "CD133 expression in oral lichen planus correlated with the risk for progression to oral squamous cell carcinoma," Annals of Diagnostic Pathology, vol. 17, no. 6, pp. 486-489, 2013.

[28] W. Liu, L. Wu, X.-M. Shen et al., "Expression patterns of cancer stem cell markers ALDH1 and CD133 correlate with a high risk of malignant transformation of oral leukoplakia," International Journal of Cancer, vol. 132, no. 4, pp. 868-874, 2013.

[29] E. Irollo and G. Pirozzi, "CD133: to be or not to be, is this the real question?" American Journal of Translational Research, vol. 5, no. 6, p. 563, 2013.

[30] P. Jayasooriya, C. Fernando, A. Suraweera, and U. Dissanayake, "Stem cell markers as a resource to predict prognosis of betel quid induced oral squamous cell carcinoma: an immunohistochemical investigation," Stomatological Disease and Science, vol. 1, pp. 29-34, 2017.

[31] M. Y-P. Kuo, S-J. Cheng, H-M. Chen, S-H. Kok, L-J. Hahn, and C-P. Chiang, "Expression of CD44s, CD44v5, CD44v6 and CD44v7-8 in betel quid chewing-associated oral premalignant lesions and squamous cell carcinomas in Taiwan," Journal of Oral Pathology \& Medicine, vol. 27, no. 9, pp. 428-433, 1998.

[32] W. Li, B. Li, R. Wang, D. Huang, W. Jin, and S. Yang, "SOX2 as prognostic factor in head and neck cancer: a systematic review and meta-analysis," Acta Oto-Laryngologica, vol. 134, no. 11, pp. 1101-1108, 2014.

[33] D. R. Camisasca, M. A. N. C. Silami, J. Honorato, F. L. Dias, P. A. S. d. Faria, and S. d. Q. C. Lourenço, "Oral squamous cell carcinoma: clinicopathological features in patients with and without recurrence," ORL, vol. 73, no. 3, pp. 170-176, 2011.

[34] N.-C. Lin, J.-T. Hsu, and K.-Y. Tsai, "Survival and clinicopathological characteristics of different histological 
grades of oral cavity squamous cell carcinoma: a singlecenter retrospective study," PLoS One, vol. 15, no. 8, Article ID e0238103, 2020.

[35] M. Zhang, T. Song, L. Yang et al., "Nestin and CD133: valuable stem cell-specific markers for determining clinical outcome of glioma patients," Journal of Experimental \& Clinical Cancer Research, vol. 27, no. 1, p. 85, 2008.

[36] D. Si, F. Yin, J. Peng, and G. Zhang, "High expression of CD44 predicts a poor prognosis in glioblastomas," Cancer Management and Research, vol. 12, pp. 769-775, 2020.

[37] R. Wachowiak, M. Krause, S. Mayer et al., "Increased L1CAM (CD171) levels are associated with glioblastoma and metastatic brain tumors," Medicine, vol. 97, no. 38, Article ID e12396, 2018.

[38] W. Yu, X. Ren, C. Hu et al., "Glioma SOX2 expression decreased after adjuvant therapy," BMC Cancer, vol. 19, no. 1, pp. 1087-1089, 2019. 